\title{
Quintessential holography
}

\author{
Pedro F. González-Díaz \\ Colina de los Chopos, Centro de Física "Miguel A. Catalán," Instituto de Física Fundamental, \\ Consejo Superior de Investigaciones Científicas, Serrano 121, 28006 Madrid, Spain
}

(Received 17 March 2011; published 1 August 2011)

\begin{abstract}
By using the Bousso geometrical covariant procedure we consider in this paper the holographic principle as applied to asymptotic Friedmann-Robertson-Walker space-times whose vacuum dark energy is due to the presence of a quintessential dark and phantom-energy scalar field with constant equation of state $p=\omega \rho$, both for $w>-1$ and $w<-1$. We have obtained the positions of the optimal screen for each case, deriving for them moreover expressions for the future event horizon and hence holographic dark-energy models of the Li and Huang and Li types, which are compared with the most consistent results derived from the covariant Bousso procedure. It is also seen that one of the two obtained holographic screens is placed on the big rip hypersurface in the phantom-energy case, both for the covariant holographic formalism and holographic phantom models. Consistency of fundamental theories is analyzed in the light of the largest physical regions allowed by the existence of a holographic screen in the future.
\end{abstract}

DOI: 10.1103/PhysRevD.84.044004

PACS numbers: 04.20.Jb, 98.80.Jk

\section{INTRODUCTION}

The holographic principle discovered by 't Hooft asserts [1-3] that all the information contained in some bounded region of space can be faithfully given as a hologram on the boundary of that region, storing at most 1 degree of freedom per Planck area. The holographic principle has become to be of a vital importance in modern particle and cosmic theories that use extra dimensions [4], in particular, those theories which are based on the so-called AdS/CFT or even the dS/CFT correspondence [5], and in those theoretical models of space-time that lead to the existence of an entropy bound [6] or a quantization of the mass such as it happens in black holes, where $[7,8] M \propto \sqrt{n} M_{p}$, with $M_{p}$ being the Planck mass. Also of a great theoretical interest is the connection between space-time holography and the now very popular $\mathrm{M}$ theory of which current versions of string theories are nothing but effective descriptions [9]. In this paper we shall consider how the holographic principle applies to those space-times that describe accelerating universes driven by a simple quintessential dark-energy scalar field with constant equation of state, $p=\omega \rho$, in the nonphantom case, where $1 / 3>\omega>-1$, and in the phantom case, where $w<-1$, using the seminal procedure devised by Bousso [10], which is confronted to the corresponding description provided by holographic dark-energy models [11]. Our analysis appears to be of interest in at least two different respects. On the one hand, dark energy, which makes up to 70 percent of the total energy of the universe, ought to contain a large amount of the relevant degrees of freedom and hence, in order to constraint the state equation for darkenergy, it should be important to investigate whether such degrees of freedom are or not projected on the same boundary surfaces as those characterizing the remaining nonvacuum energy. On the other hand, it has been pointed out [11-14] that an accelerating universe would show a future event horizon (see however $[15,16]$ ) which prevented formulating any theory which, such as it happens with any string or quantum-gravity theory, is based on the existence of a $S$ matrix at infinite distances. The emergence of an event horizon at the future, which would behave like a holographic screen, would make the above problem even more acute. Our simplifying assumption for a constant $w$ may actually require significant fine tuning of the quintessence or phantom potential [17]. At the end of the paper I will briefly discuss the possible impact that one could expect on the results of the paper from including a variable equation of state in a convenient tracked model.

This paper can be outlined as follows. Sec. II contains quintessential dark-energy solutions for the three types of spatial geometry which could still be present in an accelerating universe in the case that the parameter of the equation of state $w>-1$. In Sec. III the covariant Bousso formalism is used to derive the ultimate holographic optimal screens corresponding to the solutions obtained in Sec. II. Sec. IV contains a discussion of covariant holography for a general phantom-energy scenario. The dark and phantom-energy holographic models for flat and nonflat geometries are constructed in Sec. V in order to insert holographic screens in terms of the respective future event horizon, following the Li [11] and Huang-Li [18] models, or in terms of the horizon at the big rip, following Ref. [19]. Finally, in Sec. VI we conclude and add some comments aiming at comparing the holographic darkenergy models with the quintessential Bousso holography and discussing how all such models adapt themselves to the fundamental physical theories based on the definition of an $S$ matrix. Also included in that section is some preliminary 
material on variable $-w$ holography for a most interesting quantum model.

\section{THE SPACE-TIME OF A UNIVERSE FILLED WITH DARK ENERGY}

The problem of dark energy is possibly the most challenging one of present-day physics. In the case that dark energy be originated from the existence of a positive cosmological constant, the universe would asymptotically tend to de Sitter space, whose holographic properties have already been extensively studied [20]. If dark energy has a dynamical content and corresponds to a so-called quintessence field with either constant [21] or time-dependent [22] parameter of the equation of state, then a plethora of spacetime structures for the universe would arise whose holographic properties have not been considered yet. Thus, we shall restrict ourselves in this paper to consider the holographic principle in the asymptotic Friedmann-RobertsonWalker (FRW) space-times whose vacuum dark energy is due to the presence of a quintessential scalar field with constant parameter, $\omega$, for its equation of state $p=\omega \rho$ [21]. It is well-known that the quintessence field $\phi$ is a slowly-varying field whose energy density and pressure are generally defined by [20]

$$
\begin{aligned}
& 8 \pi G \rho=\frac{1}{2} \dot{\phi}^{2}+V(\phi) \\
& 8 \pi G p=\frac{1}{2} \dot{\phi}^{2}-V(\phi),
\end{aligned}
$$

with $V(\phi)$ the quintessential potential, which must satisfy some dynamical and observational constrains [23], and the overhead dot means $t$-time derivative.

An accelerating regime for the universe takes only place within the interval $-1 / 3>\omega>-1$, for which the deceleration parameter becomes negative. Most recent observational constraints for the parameter $\omega$ [23] restrict this parameter to be $\omega \leq-2 / 3$. Therefore, we shall consider in what follows a FRW universe endowed with a quintessence scalar field defined by Eqs. (2.1) and (2.2) in the above accelerating interval for $\omega$, specializing in the particular value $\omega=-2 / 3$. The case $\omega=-1$ corresponds to de Sitter space and will not be considered in this paper. The Friedmann equations for a FRW universe with Lorentzian signature are given by [24]

$$
\begin{aligned}
& \ddot{a}=-\frac{4 \pi G(1+3 \omega)}{3 \alpha} a^{-(2+3 \omega)} \\
& \frac{\dot{a}^{2}}{a^{2}}=\frac{8 \pi G}{3 \alpha} a^{-3(1+\omega)}-\frac{1}{R^{2} a^{2}},
\end{aligned}
$$

where $a=a(t)$ is the scale factor, $\dot{a}$ its $t$-time derivative, $\alpha$ is an arbitrary integration constant which would be related to the cosmological constant $\Lambda$ by $\alpha=8 \pi G / \Lambda$ in case that $\omega=-1$, and $R^{-2}$ is the spatial curvature.
The measured angular position of the first acoustic peak on the spectrum of CMB anisotropies [25] clearly points to a universe which is spatially flat, i.e. $R^{-2}=0$, at a high confidence level. In this observationally most favored case the scale factor for any $\omega$ within the interval $-1 / 3>$ $\omega>-1$ is given by [24]

$$
a(t)=\left(\frac{6 \pi G(1+\omega)^{2}}{\alpha}\right)^{1 /[3(1+\omega)]} t^{2 /[3(1+\omega)]},
$$

where we have disregarded an integration constant in the right hand side which corresponded to the initial value of the scale factor. That constant is not going to play any role in the analysis to follow for a nonphantom case when large values of time $t$ are associated with the future holographic screens. Now, in order to investigate the holographic properties of the considered space-times it is convenient to reexpress solution (2.5) in terms of the conformal time

$\eta=\int \frac{d t}{a(t)}=3\left(\frac{6 \pi G}{\alpha}\right)^{-1 /[3(1+\omega)]} \frac{[(1+\omega) t]^{(1+3 \omega) /[3(1+\omega)]}}{1+3 \omega}$

(note that $0<\eta<\infty$ for $\omega>-1 / 3$, and $0>\eta>-\infty$ for $\omega<-1 / 3)$, and hence we obtain for the scale factor

$$
a(\eta)=\left(\frac{8 \pi G}{3 \alpha}\right)^{1 /(1+3 \omega)}\left(\frac{\eta(1+3 \omega)}{2}\right)^{2 /(1+3 \omega)} .
$$

It is worth remarking that Eq. (2.7) is consistently the same as that was obtained by Bousso [10] for a flat FRW universe with perfect-fluid equation of state $p=\gamma \rho$, in the interval $-1 / 3<\gamma \leq 1$, unless for the $\alpha$-dependent prefactor.

In spite of the above alluded opinion that the spatially flat geometry is by much the most favored case, The case of a universe with a nonflat spatial curvature cannot be still fully discarded, neither for closed universes [26-28] in case of a small positive curvature, nor when $R^{-2}=-1$ because all current data are very near to the dividing borderline between positive and negative very small values of the spatial curvature [29]. We have been not able to obtain solutions for a generic $\omega$ in closed form in the spatially closed and open cases. We shall restrict then ourselves in what follows to the solutions that correspond to particular values of the parameter $\omega$. For the spatially closed geometry, $R^{-2}=+1$, the most general solution in terms of the FRW time $t$ for the simple case $\omega=-2 / 3$ can be written as [24]

$$
a(t)=\frac{2 \pi G}{3 \alpha} t^{2}+C_{1} t+\frac{3 \alpha}{8 \pi G}\left(1+C_{1}^{2}\right) .
$$

Without loss of generality one can choose the integration constant $C_{1}$ to be zero. We then obtain for the solution

$$
a(t)=\frac{2 \pi G}{3 \alpha} t^{2}+\frac{3 \alpha}{8 \pi G} .
$$

In this case the conformal time is given by 


$$
\eta=2 \arctan \left(\frac{4 \pi G}{3 \alpha} t\right)
$$

where an integration constant, namely, $K$, has been absorbed in the conformal time so that $\eta=\eta^{\prime}-K$. $\eta$ in this expression will vary between $\eta=0$ and $\eta=+\pi$. In terms of the conformal time given by $0>\eta=$ $-9 \alpha /(2 \pi t)>-\infty$, we get for the scale factor

$$
a(\eta)=\frac{3 \alpha}{8 \pi G \cos ^{2}(\eta / 2)} .
$$

In this case the holographic analysis that we shall carry on the situation where $\omega=-2 / 3$ will represent all other possible situations that describe accelerating closed universes and that therefore will not be explicitly considered in this work.

On the other hand, for $\omega=-1 / 3$ in the closed case, we generally obtain

$$
a(t)=C_{1} t+C_{2},
$$

where $C_{1}$ and $C_{2}$ are integration constants. In this case, the conformal time is given by $\ln \left(C_{2}\right) / C_{1} \leq \eta=$ $\ln (a(t)) / C_{1} \leq \infty$, and therefore $a(\eta)=C_{3} \exp \left(C_{1} \eta\right)$, with $C_{3}$ still another integration constant.

Finally, we shall briefly concentrate on the case $R^{-2}=-1$ that corresponds to an open universe for particular values of $\omega$. Again for $\omega=-2 / 3$, the most general solution to the Friedmann equations in terms of the FRW time $t$ is

$$
a(t)=\frac{2 \pi G}{3 \alpha} t^{2}+C_{1} t-\frac{3 \alpha}{8 \pi G}\left(1-C_{1}^{2}\right) .
$$

Again the choice $C_{1}=0$ can be done without any loss of generality to get

$$
a(t)=\frac{2 \pi G}{3 \alpha} t^{2}-\frac{3 \alpha}{8 \pi G},
$$

where $0>t>-3 \alpha /(4 \pi G)$. Hence,

$$
0<\eta=-2 \operatorname{arctanh}\left(\frac{4 \pi G t}{3 \alpha}\right)<\infty
$$

and, finally,

$$
a(\eta)=-\frac{3 \alpha}{4 \pi G \tanh ^{2}(\eta / 2)} .
$$

Open solutions for other values of $\omega$ within the interval $-1<\omega<-1 / 3$ would all show similar holographic properties and therefore will not be discussed here. For the sake of completeness, we shall now very briefly summarize the open solution for the uniform universe $\omega=-1 / 3$. It reads

$$
a=\sqrt{1+\frac{8 \pi G}{3 \alpha}} t+\kappa_{1}=\kappa_{2} \exp \left(\sqrt{1+\frac{8 \pi G}{3 \alpha} \eta}\right),
$$

with $\kappa_{1}$ and $\kappa_{2}$ being integration constants, and

$$
\eta=\frac{\ln [a(t)]}{\sqrt{1+\frac{8 \pi G}{3 \alpha}}},
$$

which holds in the interval $\ln \left(\kappa_{1}\right) /\left[\sqrt{1+\frac{8 \pi G}{3 \alpha}}\right] \leq \eta<\infty$.

\section{COVARIANT BOUSSO HOLOGRAPHY IN ACCELERATING QUINTESSENTIAL UNIVERSES}

In order to investigate the holographic properties of the space-times considered in Sec. II, we shall next follow the formalism devised by Bousso for general cosmological space-times [10]. Thus, starting with the Penrose-Carter conformal diagrams constructed for our asymptotic spacetimes, we will identify the embedded holographic hypersurfaces (screens or preferred screens) on which all the information contained in the bulk space with an area rate at most of a degree of freedom per Planck area [1]. We thus first slice the space-times into a family of null hypersurfaces and identify the null projections that go along either past- or future-directed light cones, centered at the origin of the FRW radial coordinate, $r=0$, and are parametrized by time. We then proceed following the recipe provided by Bousso [10].

The Penrose-Carter diagrams will be constructed following the prescription provided by Hawking and Ellis [30] by which the finite conformal diagrams for FRW spaces are immediately obtained after determining the regions of the cylindric Einstein static universe, which are conformal to the space we are dealing with. In our four-dimensional spherically symmetric space-times, every point on the resulting conformal diagrams represents a two-sphere and each diagonal line represents a light cone. Families of ascending and/or descending diagonal lines can then be generally constructed that give two independent null slicings. Apparent horizons, which would generally result in holographic hypersurfaces, will then be identified at the surfaces where the congruence of curves in the manifold ceases to expand. Such horizon will generally divide the whole space-time into normal, trapped and antitrapped regions $[10,30]$. We would finally determine all existing preferred and optimal (if any) screen hypersurfaces which are going to encode all global information in the bulk space-times. A preferred screen is that surface at every point of which the expansion of all projected null hypersurfaces vanishes [10]. If the projections of both independent pairs of orthogonal families of light rays vanish on one of such preferred screens, it becomes an optimal screen [10].

In terms of the conformal time $\eta$ the metric of a flat FRW space-time is known to be given by

$$
d s^{2}=a(\eta)^{2}\left(-d \eta^{2}+d r^{2}+r^{2} d \Omega_{2}^{2}\right),
$$

where $0<r<\infty$, and $d \Omega_{2}^{2}=d \theta^{2}+\sin ^{2} \theta d \phi^{2}$ is the metric on the unit two-sphere, with $0<\theta<\pi$ and $0<\phi<$ $2 \pi$. After introducing the usual change to the logarithm of 
advanced and retarded coordinates, i.e. coordinates $p, q$ of Ref. [30] and then the new coordinates $t^{\prime}=p+q, r^{\prime}=$ $p-q$, metric (3.1) can be reexpressed into a form which is conformal to that of Minkowski space in spherical coordinates and hence locally to that of Einstein static universe [27], that is

$$
\begin{aligned}
d s^{2}= & \frac{1}{4} a^{2} \sec ^{2}\left[\frac{1}{2}\left(t^{\prime}+r^{\prime}\right)\right] \sec ^{2}\left[\frac{1}{2}\left(t^{\prime}-r^{\prime}\right)\right]\left(-\left(d t^{\prime}\right)^{2}\right. \\
& \left.+\left(d r^{\prime}\right)^{2}+\sin ^{2} r^{\prime} d \Omega_{2}^{2}\right)
\end{aligned}
$$

with $-\pi<t^{\prime}+r^{\prime}<\pi,-\pi<t^{\prime}-r^{\prime}<\pi, r^{\prime} \geq 0$. The new coordinates $r^{\prime}$ and $t^{\prime}$ are related to the original coordinates $r$ and $\eta$ by

$$
\begin{aligned}
& \eta=\frac{1}{2} \tan \left[\frac{1}{2}\left(t^{\prime}+r^{\prime}\right)\right]+\frac{1}{2} \tan \left[\frac{1}{2}\left(t^{\prime}-r^{\prime}\right)\right] \\
& r=\frac{1}{2} \tan \left[\frac{1}{2}\left(t^{\prime}+r^{\prime}\right)\right]-\frac{1}{2} \tan \left[\frac{1}{2}\left(t^{\prime}-r^{\prime}\right)\right] .
\end{aligned}
$$

Clearly, the flat space-time filled with a quintessence field and equation of state with parameter of the equation of state in the range $-1 / 3>\omega>-1$ can be mapped into precisely that part of the cylindric Einstein static space determined by the values taken by $\eta$. In this most representative case, where $-1 / 3>\omega>-1, \eta$ lies in the interval $0>\eta>-\infty$, which corresponds to the ranges $0>t^{\prime}>-\pi$ and $0<r^{\prime}<\pi$. These ranges will be used to determine the region of the Einstein static space which is conformal to our quintessential flat space-time and hence its Carter-Penrose diagram.

In the case $R^{-2}=+1$ the space is already conformal to the Einstein static space by itself, without introducing any further geometrical manipulations. In this case, we have $0<\eta<\pi$ which implies that the quintessence closed universe for any equation of state is conformal to a cylindrical strip of Einstein static universe. For $R^{-2}=-1$ and $\omega=-2 / 3$ we also use the coordinate transformation to $r^{\prime}$ and $t^{\prime}$ given by Hawking and Ellis [30] and obtain a metric which is once again conformal to the same region of the Einstein static universe, with $\pi / 2 \geq t^{\prime}+r^{\prime} \geq-\pi / 2$ and $\pi / 2 \geq t^{\prime}-r^{\prime} \geq-\pi / 2$, as for any other value of the parameter characterizing the equation of state $p=\gamma \rho$. In Fig. 1 we represent the parts of the Einstein static cylinder which are conformal to the quintessential FRW spaces considered in this paper for $-1 / 3>\omega>=-1$ in the flat case and for $w=-2 / 3$ in the closed and open cases. We notice that only for the closed geometry our results matches those considered by Hawking and Ellis for FRW metrics [30]. For the flat and open geometries, the conformal region runs in the quintessential considered cases from $t^{\prime}=0$ to an extreme $t^{\prime}<0$, while in the FRW cases considered in Ref. [10] it runs from $t^{\prime}=0$ to $t^{\prime}>0$.

One can now give the Penrose-Carter diagrams for the three chosen cases that correspond to the flat, closed, and open accelerating universes filled with a quintessence field

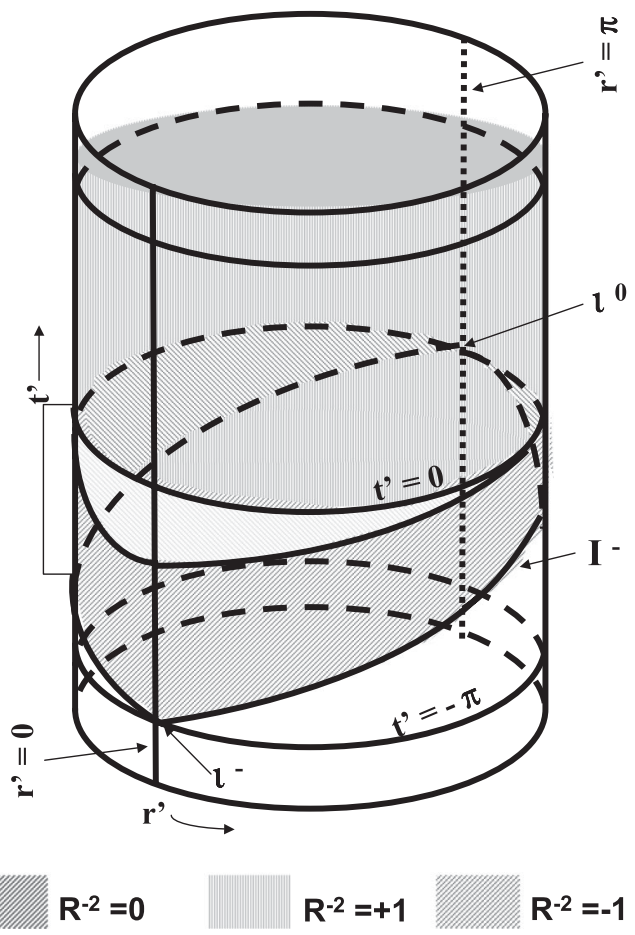

FIG. 1. The FRW space-times filled with a quintessential field with a perfect-fluid equation of state $p=\omega \rho$ is conformal to the cylindric Einstein static universe in the flat case $R^{-2}=0$ for the most interesting range $-1 / 3>\omega>-1$, and it also does at least for $w=-2 / 3$ in the cases $R^{-2}= \pm 1$. This representation matches that for usual FRW universes with $p=\Lambda=0$, for the closed case, and that for de Sitter space $(p=-\rho, \Lambda>0)$, for the open space case. The flat space case looks similar to that for the de Sitter space $(p=-\rho)$, though it covers a larger $t^{\prime}$ interval.

for an equation of state characterized by a parameter $-1 / 3>w>-1$ for the flat case and $\omega=-2 / 3$ for the closed and open cases. The diagram for the closed case is the same as that is given by Bousso for FRW universe with $p=\Lambda=0$ [10]. The diagrams for the flat and open cases are essentially the same (see Fig. 2), but they however differ from the one described by Bousso [10] and Hawking and Ellis [30].

Following then the recipe provided by Bousso [10], we can now determine the screens in the quintessential flat and open cases. The apparent horizon for any value of $\omega$ is given by $\eta=2 r /(1+3 \omega)$. The interior of these apparent horizons can now be projected along future light cones centered at $r=0$ or, by means of a spacelike projection, onto the apparent horizon. The exterior to the horizon with $\eta \leq-2 r$ can as well be projected by future light cones onto the horizon. On the other hand, the whole of the flat or open quintessential universe can also be projected along past light cones onto the past null infinity. Thus, the two holographic preferred screen hypersurfaces for the cases given in Fig. 2 are given by the apparent horizons at $\eta=$ $-2 r /(1+3 w)$ (so that $\eta$ runs from $-2 r$ to $-\infty$ ) and the 

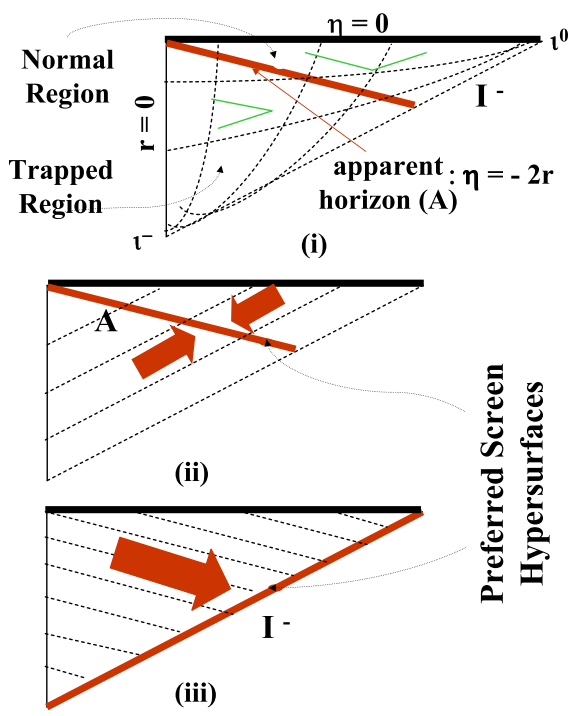

FIG. 2 (color online). Penrose-Carter diagram for a flat (or open) FRW universe filled with a quintessence scalar field with a perfect-fluid equation of state determined by a constant parameter $\omega(\omega=-2 / 3$ for the open geometry and $-1 / 3>w>-1$ for the flat geometry): (i) The apparent horizon occurs at $\eta=$ $-2 r /(1+3 w)$ and divides the space-time into a normal region and a trapped region. All the information contained in the bulk four-dimensional space-time can be projected by the Bousso's method along (ii) future light cones onto the apparent horizon, and (iii) past light cones onto past null infinity $I^{-}$. Every point on the diagrams represents a two-sphere, except at the $\iota$ 's points.

conformal past null infinity $I^{-}$. Preferred screen hypersurfaces for the closed quintessential case of course are the same as those for a FRW universe dominated by pressureless dust [10], that is two alternate apparent horizons that divide the space-time into four regions.

\section{THE PHANTOM CASE}

The cases developed here for $w=-2 / 3$ are only marginally compatible with observations and therefore not realistic. Considering the phantom case and more especially $w$ slightly lower than -1 would be much more interesting for physical cosmology [31]. In this section we shall restrict ourselves to only the general $w<-1$ phantom case for a flat geometry $R^{-1}=0$, without particularizing to any constant value of that parameter. The general scenario of a phantom universe, which in fact is of particular interest observationally [32], will be thus considered in this section. It cannot admit the kind of approximation made for the situations in which $w>-1$, where a small enough value of the initial value of the scale factor $a_{0}$ could be taken to guarantee the term where such an initial value of $a$ to be also small as this term would have the form $a_{0}^{3(1+w) / 2}$ and $w>-1$. However, if $w<-1$ then that term would get very large when $a_{0}$ is made small. In fact, the most general solution of Eqs. (2.3) and (2.4) reads

$$
\begin{aligned}
a(t) & =\left[a_{0}^{(3 / 2)(1+w)}+\frac{3}{2} C(1+w)\left(t-t_{0}\right)\right]^{2 /(3(1+w))} \\
& =T^{2 /(3(1+w))}
\end{aligned}
$$

where $C=\sqrt{6 \pi G / \alpha}$. Now, in terms of the conformal time

$$
\eta=\int \frac{d t}{T^{2 /[3(1+w)]}}=\frac{2 T^{(1+3 w) /(3(1+w))}}{C(1+3 w)},
$$

the scale factor (4.1) becomes

$$
a(\eta)=\left(\frac{C(1+3 w) \eta}{2}\right)^{2 /(1+3 w)}
$$

Unless by the involved constant the expression for $a(\eta)$ is the same as that obtained for $w>-1$ and given by Eq. (2.7), and hence it is again proportional to a flat FRW universe with a perfect-fluid equation of state $p=\gamma \rho$, in the interval $-1 / 3<\gamma \leq 1$, unless for a constant. In terms of the conformal time $\eta$ then we again can use the metric of a flat FRW space-time (3.1). However, we should note at this stage that if one wants the entire range of time $t$ to be able to run beyond the big rip barrier at $t=t_{b r}$ without inducing any negative or imaginary values of the scale factor $a(t)$, then not all values of parameter $w$ can be allowed. Only those $w$ values obeying a discrete pattern given by [19]

$$
w=-\frac{1}{3}\left(1+\frac{2 n+3}{n+1}\right), \quad n=0,1,2,3, \ldots
$$

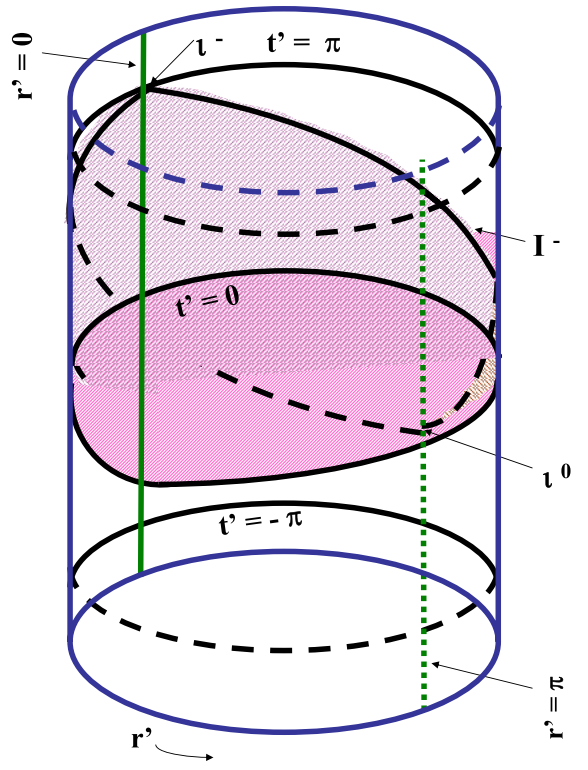

FIG. 3 (color online). The FRW space-times filled with a phantom-energy field with a perfect-fluid equation of state $p=$ $\omega \rho$ is conformal to the cylindric Einstein static universe in the flat case $R^{-2}=0$ for the most interesting range $-1>\omega>-\infty$. This flat space looks similar to that for the de Sitter space $(p=-\rho)$, though it covers a larger $t^{\prime}$ interval. 
will guarantee real and positive values for $a(t)$ from $t=0$ to $t=\infty$. Inserting then that discrete values for $w$ in the above equations for $\eta$ and $a(\eta)$, we finally have

$$
\begin{gathered}
\eta=-\frac{2(n+1)}{C(2 n+3)}\left[a_{0}^{-(1 /(2(n+1)))}-\frac{C\left(t-t_{0}\right)}{2(n+1)}\right]^{2 n+3} \\
a(\eta)=\left[-\frac{C(2 n+3)}{2(n+1)} \eta\right]^{-((2(n+1)) /(2 n+3))} .
\end{gathered}
$$

After similar manipulations to those done in the previous $w>-1$ case, we again locally obtain the metric (3.2) for the Einstein static universe, with $\eta$ and $r$ as given by Eqs. (3.3) and (3.4). In the phantom case we can readily check that $\eta$ should run in the interval starting from $\eta=-2(n+1) a_{0}^{-((2 n+3) /(2(n+1)))} / C(2 n+3) \equiv \eta_{0}<0$ for $t=t_{0}$, getting then at $\eta=0$ exactly at the big rip singularity at $t \equiv t_{b r}=t_{0}-\eta_{0}(2 n+3)$, to finally reach positive infinity as $t \rightarrow \infty$; that is we have the interval $\eta_{0}<\eta<+\infty$.
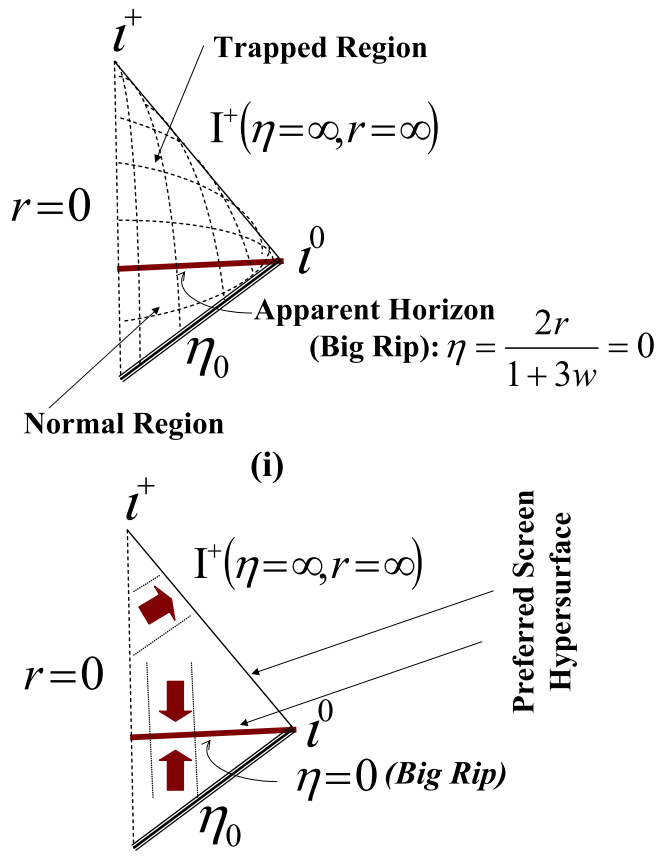

(ii)

FIG. 4 (color online). Penrose-Carter diagram for a flat FRW universe filled with a phantom-energy scalar field with a perfectfluid equation of state determined by a constant parameter $-1>$ $w>-\infty$ : (i) The apparent horizon also occurs at $\eta=$ $-2 r /(1+3 w)$ and divides the space-time into a normal region and a trapped region. All the information contained in the bulk four-dimensional space-time can be projected by the Bousso's method along (ii) using either future light cones from the normal region or past light cones from the trapped region, both onto the apparent big trip horizon, or finally by employing future light cones onto future null infinity $I^{+}$. Every point on the diagrams represents a two-sphere, except at the $\iota$ 's points.
Thus, the flat space-time filled with a phantom-energy field and equation of state $p=w \rho$, with $-\infty<w<-1$ can also be mapped into those parts of the cylindric Einstein static universe which are determined by the values pointed out above for the conformal time $\eta$. We can see that the part $0<\eta<+\infty$ of the whole interval $\eta_{0}<\eta<+\infty$ will correspond to the range $0<t^{\prime}<\pi$ and $0<r^{\prime}<\pi$, and the part $\eta_{0}>\eta>0$ will correspond to a given $w$-dependent subinterval of the range $0>t^{\prime}>$ $-\pi$ and $0<r^{\prime}<\pi$. A mapping is shown in Fig. 3 and finally leads to the Carter-Penrose diagram given in Fig. 4.

\section{HOLOGRAPHIC DARK AND PHANTOM-ENERGY MODELS}

The holographic bound on the entropy [1,6] also manifests in what has been dubbed as holographic dark-energy models [33], which are suggested to describe the properties of accelerating cosmology. Among such models, that due to Li [11] has deserved most attention. It is based on black hole physics where the short distance cutoff can be related to the infrared cutoff [34], which is taken to set the size of the observer-dependent future event horizon which makes the holographic screen. This choice has been shown to allow for a holographic dark-energy scenario and an equation of state which appear to be compatible with current observational constraints [33]. In a flat universe where dark-energy dominates, the Li model [11] predicts the relation among the Hubble parameters $H=\dot{a} / a$, the energy density $\rho \propto a^{-3(1+\omega)}$, with $\omega=p / \rho, p$ being the negative pressure, and the size of the future event horizon $R_{h}$ given by

$$
H^{2}=\frac{8 \pi G \rho}{3}=\frac{c^{2}}{R_{h}^{2}},
$$

where $a \equiv a(t)$ is the scale factor; $R_{h}=a(t) \int_{t}^{\infty} d t^{\prime} / a\left(t^{\prime}\right)$ is seen to be an ultimate region whose total energy is taken not to exceed the mass of a black hole with the same size, and $c$ is a numerical parameter of order unity which is defined as

$$
c=-\frac{2}{1+3 \omega} .
$$

Note that in our quintessence models $c>1$. Values $c<1$ should be associated with the so-called phantom models [35] that we shall consider later on. In what follows we shall investigate how the Li expression (4.1) works in each of the cases considered in Sec. II. Thus, in the general flat case with scale factor given by Eq. (2.5) we get for the size of the future event horizon

$$
R_{h}^{0}=-\frac{3(1+\omega)}{1+3 \omega} t=-\frac{2+c}{2} t .
$$

By using then the expressions for the energy density and the scale factor it is easy to show that in this case Eq. (4.1) holds and hence the Li model is satisfied. 
In cases where we consider nonflat spatially closed geometries, Huang and Li have shown [18] that holographic dark energy must be generalized to satisfy the requirement

$$
H^{2}+\frac{1}{R^{2} a^{2}}=\frac{8 \pi G \rho}{3 \alpha}=\frac{c^{2}}{R^{2} a^{2} \sin ^{2}\left(\frac{\left.\int_{t}^{\infty} \frac{d t^{\prime}}{a t^{\prime}}\right)}{R}\right)} .
$$

Let us turn now to the spatially closed geometry specializing in $\omega=-2 / 3$. We get then

$$
R_{h}^{+1}=\left[\frac{\pi}{2}-\arctan \left(\frac{4 \pi G t}{3 \alpha}\right)\right]\left(\frac{2 \pi G t^{2}}{3 \alpha}+\frac{3 \alpha}{8 \pi G}\right) .
$$

We can show that this situation satisfies the Huang-Li requirement (4.4) and hence it corresponds to a well defined holographic dark-energy model. However, when turning to the situation in which closed spatial geometries with a parameter $\omega=-1 / 3$ are considered, we have that the size of the future event horizon becomes infinite and the Huang-Li model cannot hold.

A similar failure takes place if we insist in applying Eq. (4.4) in cases of open geometries for $\omega=-2 / 3$ even if one Wick rotates the cosmic time to imaginary values so that $t \rightarrow i \tau$ and sets as the maximum value for $\tau, \tau_{\max }=$ $3 \alpha / 4 \pi G$. Nevertheless, the open geometry case for $\omega=-1 / 3$ may be a little more successful as in this case we finally get an indetermination which could be resolved by using the values of the Hubble parameter and the darkenergy density.

However, if instead of using a future event horizon, we employ the particle horizon, we will have instead of Eq. (4.4)

$$
H^{2}+\frac{1}{R^{2} a^{2}}=\frac{8 \pi G \rho}{3 \alpha}=\frac{c^{2}}{R^{2} a^{2} \sin ^{2}\left(\frac{\left.\int_{0}^{t} \frac{d t^{\prime}}{R}\right)}{R}\right)},
$$

which can be seen to be satisfied by spatially open geometry, and $\omega=-2 / 3$, for which the particle horizon is given by

$$
R_{p}^{-1}=\tanh ^{-1}\left(\frac{4 \pi G t}{3 \alpha}\right)\left(\frac{4 \pi G t^{2}}{3 \alpha}-\frac{3 \alpha}{4 \pi G}\right) .
$$

The situation for spatially open geometry and $\omega=-1 / 3$ as applied to Eq. (4.6) becomes exactly equivalent to similar situation for open geometry when applied to Eq. (4.4). In fact, when $\omega=-1 / 3$ the parameter $c$ becomes infinity so that the last expression in the right hand side of Eq. (4.6) gets an indeterminate value, compatible with the second expression for the energy density in the same equation.

Initially, it was considered [11] that holographic phantom-energy models were not viable because they cannot satisfy Eq. (5.1) for a future event horizon whose proper size inexorably becomes infinity, what may be simply interpreted as that such an event horizon vanishes for phantom energy. Thus, instead of condition (5.1), a cosmic holographic definition such that

$$
H_{\mathrm{ph}}^{2}=\frac{\dot{a}^{2}}{a^{2}}=\frac{8 \pi G \rho}{3}=\frac{c^{2}}{R_{b r}^{2}}
$$

has been proposed [19] for phantom energy, where

$$
R_{b r}=a(t) \int_{t}^{t_{b r}} \frac{d t^{\prime}}{a\left(t^{\prime}\right)}
$$

is the proper size of the future event horizon as evaluated at the big rip time $t_{b r}$. It was seen that such a horizon satisfies Eq. (5.8) and can therefore be regarded as an appropriate holographic screen for a phantom-energy universe. Since the $\mathrm{Li}$ expression (5.1) is no longer valid for a covariant description of accelerating cosmic holography, we have in this way found precisely the two holographic screens that correspond to those encountered in the covariant treatment performed in Sec. IV, one at the big rip hypersurface and the other at the future infinity.

\section{CONCLUSIONS AND FURTHER COMMENTS}

In this paper we have considered the holography of an accelerating universe when we use for the universal spacetime that stemming from both a simple quintessence model in flat, closed, and open spatial geometries and a flat phantom-energy model for any $w$. In this way, we have made recourse to the covariant Bousso formalism [10] and the so-called holographic dark-energy models $[11,18,33]$. It would be expected that the first of such procedures be less speculative than the second one. In fact, both for open and flat geometries the Bousso formalism leads to two distinct holographic preferred screened hypersurfaces, at the apparent horizons at $\eta=2 r /(1+3 \omega)$ and at the conformal past null infinity $I^{-}$for the dark-energy case or $I^{+}$ for the phantom-energy scenario. When compared with the results derived using the $\mathrm{Li}$ and Huang-Li methods (where the holographic screen is generally placed just at a finite future event horizon), we see that these results are quite more general and allow for the definition of fundamental theories based on the existence of a $S$ matrix at infinite distances, at least as one approaches the past or future infinity. The emergence of an event horizon at the finite future, which would behave like a holographic screen in holographic dark or phantom-energy models, would make however the above problem of the existence of a $S$ matrix at infinite distance [12-14] even more acute. The case for closed geometries for $w>-1$ is quite more contrived and both in Bousso and Huang-Li formalisms the problem of the $S$ matrix looks without any reasonable outcome. Whereas the Bousso method predicts two alternate apparent horizons dividing the space-time in four regions, the Huang-Li method also predicts a future event horizon. Thus, even though observational data [26-28] still leave 
some room for the current universe to be closed, it appears that such a possibility poses very difficult theoretical problems and cannot be reconciled with the very concept of physical holography.

One point concerning all these finite future horizons is worth pointing to. It is that the existence of the above horizons makes it possible for the universe to develop an entanglement entropy [36] between the regions before and after the horizons, which would ultimately amount to quantum-mechanical nonlocality between the point like objects physically traveling finite distances characterizing the region before the horizons and those point like objects able to physically travel even infinite distances after the horizons. Thus, quantum-mechanical versions of fundamental theories could always be formulated basing on the existence of $S$ matrices defined at infinite distances after the horizons.

Before closing up I will briefly discuss the potential impact that a variable equation of state may have on the above results of this paper. For that aim to be partly fulfilled, I will consider a singularity-free phantom quantum model [37] which fits well current requirements and observations, corresponding to a variable $w$ parameter given by

$$
w(t)=-\left(1+\frac{8 \pi G \epsilon_{\mathrm{Ent}}}{3\left(H_{0}+4 \pi G \epsilon_{\mathrm{Ent}} t\right)^{2}}\right)
$$

and a scale factor

$$
a(t)=a_{0} e^{H_{0} t+2 \pi G \epsilon_{\mathrm{Ent}} t^{2}},
$$

with $H_{0}$ plying the role of a cosmological constant and $\epsilon_{\text {Ent }}$ being a constant cosmic entanglement energy density. If we tried to adjust this model to the Li's holographic description for dark energy [11], then we had to define the corresponding holographic model by means of the relation

$$
H^{2}=\frac{8 \pi G \rho}{3}=4 \pi G \epsilon_{\mathrm{Ent}} \mu(t)^{2} \ln \left(8 G \epsilon_{\mathrm{Ent}} R_{h}^{2}\right),
$$

where the future event horizon is given by

$$
R_{h}=\frac{\exp \left(x^{2}\right)}{\sqrt{8 G \epsilon_{\text {Ent }}}}[1-\Phi(x)],
$$

with $\Phi(x)$ the probability integral [38],

$$
x=\frac{H_{0}}{\sqrt{8 \pi G \epsilon_{\mathrm{Ent}}}}+\sqrt{2 \pi G \epsilon_{\mathrm{Ent}}},
$$

and

$$
\mu^{-2}=1+3(1+w(t)) \ln \left[1-\Phi\left(-\frac{1}{1+w(t)}\right)\right]
$$

It is readily seen that this holographic formulation does not satisfy the original Li Eq. (5.1). A better and quite simpler description for a holographic phantom-energy model can now be introduced by using the definition of the energy density of the model which reads [37]

$$
\rho=\frac{3 H^{2}}{8 \pi G}=\frac{3}{8 \pi G R_{H}^{2}},
$$

with $R_{H}$ the proper radius of the Hubble horizon, quite a more natural holographic screen than the event and particle horizons. Moreover, in terms of the error function $\operatorname{erf}(x)$ the conformal time of the considered model would read

$$
\eta=P_{0} \operatorname{erf}\left(\frac{h_{0}+4 \pi G \epsilon_{\mathrm{Ent}} t}{\sqrt{8 \pi G \epsilon_{\mathrm{Ent}}}}\right)
$$

in which

$$
P_{0}=\frac{\pi \exp \left(\frac{H_{0}^{2}}{8 \pi G \epsilon_{\mathrm{Ent}}}\right)}{4 \sqrt{2 \pi G \epsilon_{\mathrm{Ent}}} a_{0}} .
$$

It follows that $0<\eta \leq P_{0}$; i.e. the considered tracking phantom model is conformal to the cylindric Einstein universe just on the lower part defined by $0>t^{\prime}>-\pi$, $0<r^{\prime}<\pi$ on Fig. 3. In addition, the holographic apparent horizon can now be described on the Carter-Penrose diagram by

$$
\eta=\frac{r}{1+\frac{4 \pi G \epsilon_{\mathrm{Ent}}}{\left(H_{0}+4 \pi G \epsilon_{\mathrm{Ent}} t\right)^{2}}} .
$$

Thus, this horizon occurs on $\eta<r$ (i.e. $t^{\prime}<r^{\prime}$ ) as $t \rightarrow 0$ and on $\eta=r$ (i.e. $t^{\prime}=r^{\prime}$ ) as $t \rightarrow \infty$.

\section{ACKNOWLEDGMENTS}

The author thanks Carmen L. Sigüenza of the Estación Ecológica de Biocosmología, Medellín, for useful discussions and technical help. This work was supported by MICINN under Research Project No. FIS2008-06332.
[1] G. 't Hooft, arXiv:gr-qc/9310006; L. Susskind, J. Math. Phys. (N.Y.) 36, 6377 (1995).

[2] See: http://www.damtp.ac.uk/research/gr/public/holo/.
[3] Plato, Republic edited by Stephen Watt (Wordsworth Editions, London, 1997), p. XIV.

[4] P. Midodashvili, arXiv:hep-th/0602237v1. 
[5] O. Aharony, S. S. Gubser, J. Maldacena, H. Oogun, and Y. Oz, Phys. Rep. 323, 183 (2000); B. McInnes, J. High Energy Phys. 08 (2002) 029.

[6] P. F. González-Díaz, Phys. Rev. D 27, 3042 (1983).

[7] J. D. Bekenstein, Lett. Nuovo Cimento Soc. Ital. Fis. 11, 467 (1974).

[8] C. Vaz and L. Witten, Phys. Rev. D 60, 024009 (1999).

[9] P. Horava, Phys. Rev. D 59, 046004 (1999).

[10] R. Bousso, Rev. Mod. Phys. 74, 825 (2002).

[11] M. Li, Phys. Lett. B 603, 1 (2004).

[12] W. Fischler, A. Kashani-Poor, R. McNees, and S. Paban, J. High Energy Phys. 07 (2001) 003; S. Hellerman, N. Kaloper, and L. Susskind, J. High Energy Phys. 06 (2001) 003.

[13] X.-G. He, arXiv:astro-ph/0105005.

[14] T. Banks, arXiv:hep-th/0007146.

[15] P. F. González-Díaz, Phys. Lett. B 522, 211 (2001).

[16] J. Ellis, N. E. Navromatos, and D. V. Nanopoulos, arXiv: hep-th/0105206.

[17] P. P. Avelino, A. M. M. Trindale, and P. T. P. Viana, Phys. Rev. D 80, 067302 (2009); P. P. Avelino, L. Losano, and J. J. Rodrigues, Phys. Lett. B 699, 10 (2011).

[18] Q.-G. Huang and M. Li, J. Cosmol. Astropart. Phys. 08 (2004) 002.

[19] P. F. González-Díaz, Grav. Com. 12, 29 (2006).

[20] A. Karch, J. High Energy Phys. 07 (2003) 050; M. Alishahiha, A. Karch, and E. Silverstein, J. High Energy Phys. 06 (2005) 028.

[21] R. R. Caldwell, R. Dave, and P. J. Steinhardt, Phys. Rev. Lett. 80, 1582 (1998).

[22] P. J. Steinhardt, L. Wang, and I. Zlatev, Phys. Rev. Lett. 82, 896 (1999).
[23] W. M. Wood-Vasey et al., Astrophys. J. 666, 694 (2007); Y. Wang and L. Xu, Phys. Rev. D 81, 983523 (2010).

[24] P. F. González-Díaz, Phys. Rev. D 62, 023513 (2000).

[25] P. de Bernardis, Nature (London) 404, 955 (2000).

[26] A. G. Riess, Astrophys. J. 607, 665 (2004).

[27] C. L. Bennett et al., Astrophys. J. Suppl. Ser. 148, 1 (2003).

[28] M. Tegmark et al., Phys. Rev. D 69, 103501 (2004).

[29] J. Dossett, M. Ishak, J. Holdenhauer, Y. Gong, and A. Wang, J. Cosmol. Astropart. Phys. 04 (2010) 022.

[30] S. Hawking and G. F. R. Ellis, The Large Scale Structure of Space-Time (Cambridge University Press, Cambridge, England, 1975).

[31] R. R. Caldwell, M. Kamionkowski, and N. N. Weinberg, Phys. Rev. Lett. 91, 071301 (2003).

[32] A. Melchiorri, L. Mersini, C. J. Odman, and M. Trodden, Phys. Rev. D 68, 043509 (2003).

[33] M. Li, X.-D. Li, S. Wang, and X. Zhang, J. Cosmol. Astropart. Phys. 06 (2009) 036.

[34] A. Cohen, D. Kaplan, and A. Nelson, Phys. Rev. Lett. 82, 4971 (1999).

[35] R. R. Caldwell, Phys. Lett. B 545, 23 (2002).

[36] R. Müller and C.O. Lousto, Phys. Rev. D 52, 4512 (1995).

[37] P.F. González-Díaz and A. Rozas-Fernádez, Classical Quantum Gravity 25, 175023 (2008).

[38] I. S. Gradshteyn and I. M. Ryzhik, Table of Integrals, Series, and Products (Academic, New York, 1980); M. Abramowitz and I. A. Stegun, Handbook of Mathematical Functions (Dover, New York, 1972). 